\title{
THERMAL NOISE IN DOUBLE INJECTION DIODES OPERATING IN THE INSULATOR REGIME
}

\author{
R. J. J. ZIJLSTRA and A. GISOLF \\ Fysisch Laboratorium der Rijksuniversiteit, Utrecht, Netherlands \\ (Received 15 November 1971 ; in revised form 15 February 1972)
}

\begin{abstract}
Thermal noise calculations, based on the Langevin procedure, are given for a double injection diode operating in the insulator regime. The frequency range is restricted to frequencies smaller than the reciprocal free carrier transit times. It turns out that at high frequencies the spectral density of the ac short circuit current fluctuations can be described by Nyquist's formula. At low frequencies an additional term, proportional to $\left(1+\omega^{2} \tau^{2}\right)^{-1}$ was found, where $\tau$ is the hole life time. The low frequency thermal noise level may be some orders of magnitude larger than the level at high frequencies. It is shown that in general the frequency dependent term will be masked by generation recombination noise. A special case is indicated where those contributions become comparable.
\end{abstract}

\section{INTRODUCTION}

Since noise data on double injection diodes operating in the insulator regime[1] become available [2], it is worthwhile to calculate the thermal noise in such devices. Current noise due to recombinations has been calculated before $[4,5]$.

We consider an insulator, provided with a holeinjecting (anode) and electron-injecting (cathode) contact. We will assume that the current is predominantly determined by carriers injected at the contacts and that the latter do not impose any constraint on current flow. In addition we assume that free carrier recombination takes place through localized Shockley-Read centres and that recombination of holes is monomolecular. The recombination mechanism involves the capture of a hole and subsequent recombination with an electron. The latter is supposed to be a very rapid process so that recombination is determined by the hole life time. Centres are neutral when electron occupied. Generations of free electrons and holes are ignored.

We have a planar geometry in mind, i.e. the contact spacing is small with respect to the crosssectional dimensions of the diode so that a one dimensional treatment is warrented.

In order to calculate the current and voltage fluctuations, we avail ourselves of the Langevin procedure. This implies that the macroscopic equations describing particle current flow, and the rate equations of electrons and holes are extended by so called Langevin source functions which formally describe the fluctuation processes involved.

Neglecting diffusion the equations for particle current flow, Poisson's Law, and the hole rate equation, for this case read:

$$
\begin{gathered}
J_{p}=q \mu_{p} p E+H_{p}(x, t) \\
J_{n}=q \mu_{n} n E+H_{n}(x, t) \\
J=J_{p}+J_{n} \\
\frac{\partial E}{\partial x}=\frac{q}{\epsilon \epsilon_{0}}(p-n) \\
\frac{\partial p}{\partial t}=-\frac{p}{\tau}-\frac{1}{q} \frac{\partial J_{p}}{\partial x}+H_{g r}(x, t)
\end{gathered}
$$

where: $\quad J_{p}=$ hole current flow

$$
J_{n}=\text { electron current flow }
$$

$n=$ free electron density

$p=$ free hole density

$E=$ electric field strength

$\epsilon_{0}=$ dielectric constant

$-q=$ electronic charge

$\mu_{n, p}=$ electron, hole mobility, assumed independent of $E$

$\tau=$ free hole life time

$H_{p}=$ stochastic source function for thermal noise of holes 


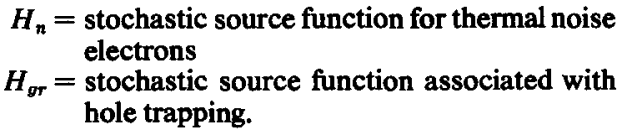

Note that because of the assumptions made, we do not need the rate equation for free electrons, nor that for electrons in the recombination centres.

We will only consider frequencies small with respect to the reciprocal electron and hole transit times. This implies that displacement current can be ignored and hence

$$
\frac{\partial J}{\partial x}=-\epsilon \epsilon_{0} \frac{\partial^{2} E}{\partial x \partial t} \approx 0 .
$$

The source functions $H_{p}$ and $H_{n}$ formally describe the random motion of electrons and holes induced by scattering processes [6]. For the cross correlation spectral densities $S_{H_{p}}$ and $S_{H_{n}}$ we write

$$
\begin{aligned}
& S_{H_{p}}\left(x_{1}, x_{2}, f\right)=4 q^{2} D_{p} p_{0} \frac{\delta\left(x_{1}-x_{2}\right)}{A} \\
& S_{H_{n}}\left(x_{1}, x_{2}, f\right)=4 q^{2} D_{n} n_{0} \frac{\delta\left(x_{1}-x_{2}\right)}{A}
\end{aligned}
$$

where $D_{n}$ and $D_{p}$ are the diffusion constants for electrons and holes respectively, $A$ is the crosssectional area, $\delta$ is the Dirac delta function, and the subscript 0 indicates steady state values.

\section{CALCUlations Of THERMAL NOISE}

By eliminating $n$ from equations (3) and (4) we can write for $p$

$$
p=\frac{J-H_{n}-H_{p}+\epsilon \epsilon_{0} \mu_{n}(\partial E / \partial x) E}{q E\left(\mu_{n}+\mu_{p}\right)} .
$$

Substituting equation (1) into equation (5) and using equation (9) yields:

$$
\begin{aligned}
& \frac{J-H_{n}-H_{p}}{E}+\tau \frac{\partial}{\partial t}\left(\frac{J-H_{n}-H_{p}}{E}\right)= \\
& -\epsilon \epsilon_{0} \mu_{n} \frac{\partial E}{\partial x}-b \frac{\partial}{\partial x}\left(E \frac{\partial E}{\partial x}\right)+\tau\left(\mu_{p} \frac{\partial H_{n}}{\partial x}-\mu_{n} \frac{\partial H_{p}}{\partial x}\right)+ \\
& +\tau q\left(\mu_{n}+\mu_{p}\right) H_{g r}
\end{aligned}
$$

where $b=\mu_{n} \mu_{p} \epsilon \epsilon_{0} \tau$.

Following Driedonks [5], we will assume that for the diode operating in the insulator regime, the first term on the right hand side of equation (10) can be ignored. This implies that space charge is neglected.

Linearizing equation (10) by considering fluctuations around a steady state gives

$$
J_{0}=-b E_{0} \frac{\mathrm{d}}{\mathrm{d} x}\left(E_{0} \frac{\mathrm{d} E_{0}}{\mathrm{~d} x}\right)
$$

and

$$
\begin{aligned}
& \left(\Delta J-H_{n}-H_{p}\right) E_{0}-J_{0} \Delta E+\tau \frac{\partial}{\partial t}\left[\left(\Delta J-H_{n}-H_{p}\right) E_{0}-J_{0} \Delta E\right]= \\
& =-b E_{0}{ }^{2} \frac{\partial^{2}}{\partial x^{2}}\left(\Delta E E_{0}\right)+\tau E_{0}{ }^{2}\left[\mu_{p} \frac{\partial H_{n}}{\partial x}-\mu_{n} \frac{\partial H_{p}}{\partial x}+q\left(\mu_{n}+\mu_{p}\right) H_{a r}\right]
\end{aligned}
$$

The use of equations (7) and (8) can be justified by the fact that in the ohmic regime this formula leads to the familiar Nyquist expression for thermal noise if the Einstein relations are valid, i.e. if $q D_{n}=\mu_{n} k T$ and $q D_{p}=\mu_{p} k T$, where $k$ is Boltzmann's constant and $T$ is the device temperature. Also in our case we will assume that the Einstein relations hold. This implies that the velocity distribution is maxwellian, despite the applied electric field.

In the Langevin source functions electrons and holes are treated as independent. Correlation in their densities is taken into account by using the equations (1)-(5). where steady state values are denoted by subscript 0 . Making a Fourier analysis of equation (12), considering truncated time samples of length $T$, one finds

$$
\begin{aligned}
& -b E_{0}^{2} \frac{\partial^{2}}{\partial x^{2}}\left(\tilde{E} E_{0}\right)+J_{0}(1+i \omega \tau) \tilde{E}= \\
& E_{0}(1+i \omega \tau)\left(\tilde{J}-\tilde{H}_{n}-\tilde{H}_{p}\right)- \\
& -\tau E_{0}^{2}\left[\mu_{p} \frac{\partial \tilde{H}_{n}}{\partial x}-\mu_{n} \frac{\partial \tilde{H}_{p}}{\partial x}+q\left(\mu_{n}+\mu_{p}\right) \tilde{H}_{v r}\right]
\end{aligned}
$$

where Fourier transforms of corresponding 
quantities are denoted by tilde, $i$ is the imaginary unit, and $\omega$ the circular frequency. Upon integration of equation (13) over $x$ using partial integrations for the first term on the left hand side one finds

$$
\begin{aligned}
& \int_{0}^{L} 2 b \tilde{E} E_{0} \frac{\mathrm{d}}{\mathrm{d} x}\left(E_{0} \frac{\mathrm{d} E_{0}}{\mathrm{~d} x}\right) \mathrm{d} x+J_{0}(1+i \omega \tau) \tilde{V} \\
& =V_{0}(1+i \omega \tau) \tilde{J}+\int_{0}^{L}(1+i \omega \tau) E_{0}\left(\tilde{H}_{n}+\tilde{H}_{p}\right) \mathrm{d} x+ \\
& +\int_{0}^{L} \tau E_{0}^{2}\left[\mu_{p} \frac{\partial \tilde{H}_{n}}{\partial x}-\mu_{n} \frac{\partial \tilde{H}_{p}}{\partial x}+q\left(\mu_{n}+\mu_{p}\right) \tilde{H}_{g r}\right] \mathrm{d} x
\end{aligned}
$$

where the usual boundary conditions $E(0)=E(L)$ $=0$ are used, $L=$ contact spacing, $V_{0}=$ d.c. applied voltage, and $\tilde{V}=$ Fourier transform of the a.c. voltage. Using equation (11) in rewriting the first term on the left hand side yields after some algebra:

$$
\begin{aligned}
& \tilde{V}=\frac{1}{J_{0}(3+i \omega \tau)}\left\{V_{0}(1+i \omega \tau) \tilde{J}\right. \\
& +\int_{0}^{L}(1+i \omega \tau) E_{0}\left(\tilde{H}_{n}+\tilde{H}_{p}\right) \mathrm{d} x+ \\
& \left.+\int_{0}^{L} \tau E_{0}^{2}\left[\mu_{p} \frac{\partial \tilde{H}_{n}}{\partial x}-\mu_{n} \frac{\partial \tilde{H}_{p}}{\partial x}+q\left(\mu_{n}+\mu_{p}\right) \tilde{H}_{g r}\right] \mathrm{d} x\right\} .
\end{aligned}
$$

Putting $\tilde{H}_{n}, \bar{H}_{p}$ and $\widetilde{H}_{g r}$ equal to zero in equation (15) one finds the already known result $[4,5]$ for the a.c. small signal admittance

$$
Y \equiv \frac{-A \tilde{J}}{\tilde{V}}=\frac{I_{0}}{V_{0}} \frac{3+i \omega \tau}{1+i \omega \tau}
$$

where $I_{0}=$ d.c. current. The $J_{0}, V_{0}$ characteristic can be calculated from equation (11) and is well known to be [1]

$$
J_{0}=-\frac{125}{18} \epsilon \epsilon_{0} \mu_{n} \mu_{p} \tau \frac{V_{0}^{3}}{L^{5}}
$$

Turning to the noise we put $\tilde{J}=0$ in equation (15). The noise sources $H_{n}, H_{p}$ and $H_{g r}$ are as- sumed to be statistically independent. The resulting noise contributions in voltage or current induced by the sources mentioned can be calculated separately. Since the $g-r$ noise has been calculated before $[4,5]$, we will omit $\tilde{H}_{g r}$ in equation (15) and only calculate the thermal noise. For the open circuit voltage fluctuations we thus find

$$
\begin{gathered}
\tilde{V}=\frac{1}{J_{0}(3+i \omega \tau)} \int_{0}^{L}\left[(1+i \omega \tau) E_{0}\left(\tilde{H}_{n}+\tilde{H}_{p}\right)+\tau E_{0}^{2}\right. \\
\left.\left(\mu_{p} \frac{\partial \tilde{H}_{n}}{\partial x}-\mu_{n} \frac{\partial \tilde{H}_{p}}{\partial x}\right)\right] \mathrm{d} x
\end{gathered}
$$

The spectral density $S_{V}$ can be found from

$$
S_{V}(f)=\lim _{T \rightarrow \infty} \frac{2}{T}\left\langle\tilde{V} \tilde{V}^{*}\right\rangle
$$

using

$$
\begin{gathered}
S_{H_{n}}\left(x_{1}, x_{2}, f\right)=\lim _{T \rightarrow \infty} \frac{2}{T}\left\langle\tilde{H}_{n}\left(x_{1}\right) \tilde{H}_{n}^{*}\left(x_{2}\right)\right\rangle \\
S_{H_{p}}\left(x_{1}, x_{2}, f\right)=\lim _{T \rightarrow \infty} \frac{2}{T}\left\langle\tilde{H}_{p}\left(x_{1}\right) \tilde{H}_{p}^{*}\left(x_{2}\right)\right\rangle \\
\lim _{T \rightarrow \infty} \frac{2}{T}\left\langle\tilde{H}_{n}\left(x_{1}\right) \tilde{H}_{p}^{*}\left(x_{2}\right)\right\rangle=0
\end{gathered}
$$

where the asterisk denotes complex conjugate quantities. From equations (18)-(22) we find for $S_{V}$

$$
\begin{aligned}
& S_{V}(f)=\frac{1}{J_{0}^{2}\left(9+\omega^{2} \tau^{2}\right)} \int_{0}^{L} \\
& \int_{0}^{L} \mathrm{~d} x_{1} \mathrm{~d} x_{2}\left[\left(1+\omega^{2} \tau^{2}\right) E_{0}\left(x_{1}\right) E_{0}\left(x_{2}\right) S_{H_{n}}\left(x_{1}, x_{2}, f\right)+\right. \\
& \quad+\left(1+\omega^{2} \tau^{2}\right) E_{0}\left(x_{1}\right) E_{0}\left(x_{2}\right) S_{H_{p}}\left(x_{1}, x_{2}, f\right)+ \\
& \quad+(1+i \omega \tau) \tau \mu_{p} E_{0}\left(x_{1}\right) E_{0}^{2}\left(x_{2}\right) \frac{\partial}{\partial x_{2}} S_{H_{n}}\left(x_{1}, x_{2}, f\right)- \\
& \quad+(1+i \omega \tau) \tau \mu_{n} E_{0}\left(x_{1}\right) E_{0}^{2}\left(x_{2}\right) \frac{\partial}{\partial x_{2}} S_{H_{p}}\left(x_{1}, x_{2}, f\right)+ \\
& \quad-i \omega \tau) \tau \mu_{p} E_{0}^{2}\left(x_{1}\right) E_{0}\left(x_{2}\right) \frac{\partial}{\partial x_{1}} S_{H_{n}}\left(x_{1}, x_{2}, f\right)+
\end{aligned}
$$




$$
\begin{aligned}
& +\tau^{2} \mu_{p}^{2} E_{0}^{2}\left(x_{1}\right) E_{0}^{2}\left(x_{2}\right) \frac{\partial^{2}}{\partial x_{1} \partial x_{2}} S_{H_{n}}\left(x_{1}, x_{2}, f\right)- \\
& -(1-i \omega \tau) \tau \mu_{n} E_{0}^{2}\left(x_{1}\right) E_{0}\left(x_{2}\right) \frac{\partial}{\partial x_{1}} S_{H_{p}}\left(x_{1}, x_{2}, f\right) \\
& \left.+\tau^{2} \mu_{n}^{2} E_{0}^{2}\left(x_{1}\right) E_{0}^{2}\left(x_{2}\right) \frac{\partial^{2}}{\partial x_{1} \partial x_{2}} S_{H_{p}}\left(x_{1}, x_{2}, f\right)\right] .
\end{aligned}
$$

Rewriting equations (7) and (8) using the Einstein relations and putting

$$
n_{0} \approx p_{0}=\frac{J_{0}}{q\left(\mu_{n}+\mu_{p}\right)} \frac{1}{E_{0}}
$$

yields

$$
S_{H_{n}}=4 k T \frac{J_{0}}{A} \frac{\mu_{n}}{\mu_{n}+\mu_{p}} \frac{\delta\left(x_{1}-x_{2}\right)}{E_{0}\left(x_{1}\right)}
$$

and

$$
S_{H_{p}}=4 k T \frac{J_{0}}{A} \frac{\mu_{p}}{\mu_{n}+\mu_{p}} \frac{\delta\left(x_{1}-x_{2}\right)}{E_{0}\left(x_{1}\right)}
$$

Using equations (25) and (26) in equation (23) and evaluating the integrals, the $3^{\text {rd }}, 4^{\text {th }}, 5^{\text {th }}$ and $7^{\text {th }}$ terms turn out to be zero. We finally find with the help of equation (11)

$$
S_{V}=4 k T \frac{1+\omega^{2} \tau^{2}}{9+\omega^{2} \tau^{2}}\left[\frac{V_{0}}{I_{0}}+\frac{\tau}{1+\omega^{2} \tau^{2}} \frac{4 L}{\epsilon \epsilon_{0} A}\right] .
$$

For the short circuit current spectral density $S_{I}=S_{V}|Y|^{2}$ we obtain

$S_{I}=4 k T \frac{I_{0}}{V_{0}}\left[1+\frac{\tau}{\left(1+\omega^{2} \tau^{2}\right)} \frac{I_{0} 4 L}{V_{0} \epsilon \epsilon_{0} A}\right]$.

\section{DISCUSSION}

At high frequencies only the first term on the right hand side of equation (28) is important and equation (28) reduces to the familiar Nyquist expression, since from equation (16) we see that for $\omega \gg 1 / \tau$

$$
\operatorname{Re}(Y)=\frac{I_{0}}{V_{0}}
$$

It should be noted that the high frequency result can be obtained directly from the current equation. Since for frequencies larger than the reciprocal free carrier common lifetime, fluctuations in the free carrier densities may be neglected, one has

$$
\frac{\Delta J}{\sigma(x)}=\Delta E(x)+\frac{H_{n}+H_{p}}{\sigma(x)}
$$

where $\sigma(x)$ is the steady state conductivity. Making a Fourier analysis and integrating over $x$ yields

$$
\tilde{J} A \frac{V_{n}}{I_{0}}=-\tilde{V}+\int_{0}^{L} \frac{\tilde{H}_{n}+\tilde{H}_{p}}{\sigma} \mathrm{d} x
$$

Considering short circuit current fluctuations we have

$$
\hat{\imath}=-\frac{I_{0}}{V_{0}} \int_{0}^{L} \frac{\tilde{H}_{n}+\tilde{H}_{p}}{\sigma} \mathrm{d} x
$$

and

$S_{I}=\frac{I_{0}^{2}}{V_{0}^{2}} \int_{0}^{L} \int_{0}^{L} \frac{S_{H_{n}}\left(x_{1}, x_{2}, f\right)+S_{H_{p}}\left(x_{1}, x_{2}, f\right)}{\sigma\left(x_{1}\right) \sigma\left(x_{2}\right)} \mathrm{d} x_{1} \mathrm{~d} x_{2}$.

Using equations (25) and (26) we find

$$
S_{H_{n}}+S_{H_{p}}=4 k T \sigma\left(x_{1}\right) \frac{\delta\left(x_{1}-x_{2}\right)}{A} .
$$

Substituting this result in equation (28e) we find

$$
S_{I}=4 k T \frac{I_{0}}{V_{0}}
$$

Note that in deriving equation $(28 \mathrm{~g})$ no specific assumptions concerning carrier injection have been made and therefore the result seems quite general. Experimental evidence in support of this result for the semiconductor regime is given by Lee and Nicolet [3].

Note also that by putting $\tilde{H}_{n}=\tilde{H}_{p}=0$ in equation (28c) directly follows

$$
Y=\frac{I_{0}}{V_{0}}
$$

The second term on the right hand side of equation (28) is important only at low frequencies. By introducing $R_{0}=V_{0} / I_{0}$ and $C_{0}=\epsilon \epsilon_{0} A / L$ one can 
rewrite equation (28) in the following form

$$
S_{I}=\frac{4 k T}{R_{0}}\left[1+\frac{4}{1+\omega^{2} \tau^{2}} \cdot \frac{\tau}{R_{0} C_{0}}\right]
$$

this form is somewhat more revealing than equation (28) in that $C_{0}$ is clearly the geometrical capacitance and $R_{0} C_{0}$ is related to the ohmic or dielectric relaxation time. Moreover it shows clearly that the second term between brackets can be several orders of magnitude larger than one.

Since the thermal noise source enters rate equation (5) through the divergence of the current, one expects a frequency dependent noise contribution of the generation-recombination type. Therefore it is surprising that such a term is absent in the ohmic regime. This becomes clear however, by inspection of equation (23). The frequency dependent contributions stem here from the $6^{\text {th }}$ and $8^{\text {th }}$ term. Now it can be shown that these contributions are nonexistent when the steady state electric field strength $E_{0}$ is space independent. This explains mathematically the difference in outcome for the ohmic and insulator regime. Apparcntly the fact that current is predominantly recombination limited in the insulator regime has something to do with it. The random thermal movement of carriers, the origin of thermal noise, gives rise to space charge fluctuations, which in the ohmic regime are relaxed through ohmic conduction only, whereas in the insulator regime this occurs partly through the much slower recombination process.

In general the frequency dependent thermal noise contribution will be masked by $g-r$ noise as we will show. Driedonks found for the short circuit current spectral density due to recombinations in a double injection diode operating in the insulator regime

$$
S_{I_{g r}}=\frac{50}{21} \frac{q\left(\mu_{n}+\mu_{p}\right)}{L^{2}} I_{0} V_{0} \frac{\tau}{1+\omega^{2} \tau^{2}} .
$$

Adding the right hand sides of equations (28) and (29) we find for the total spectral density

$$
S_{I_{\text {tot }}}=4 k T \frac{I_{0}}{V_{0}}+\frac{\tau}{1+\omega^{2} \tau^{2}}\left(C_{t h}+C_{g r}\right)
$$

where

$$
C_{t h}=\frac{I_{0}^{2} 16 k T L}{V_{0}^{2} \epsilon \epsilon_{0} A} \text { and } C_{g r}=\frac{50 q\left(\mu_{n}+\mu_{p}\right) I_{0} V_{0}}{21 L^{2}}
$$

So

$$
\frac{C_{t h}}{C_{g r}}=\frac{168 I_{0} k T L^{3}}{25 V_{0}^{3} q\left(\mu_{n}+\mu_{p}\right) \epsilon \epsilon_{0} A} .
$$

With the help of equation (17) this reduces to

$$
\frac{C_{t h}}{C_{g r}}=\frac{70}{3} \frac{L_{a}^{2}}{L^{2}}
$$

where

$$
L_{a}=\left[\frac{2 k T \mu_{n} \mu_{p} \tau}{q\left(\mu_{n}+\mu_{p}\right)}\right]^{1 / 2}
$$

is the ambipolar diffusion length. A necessary condition for neglecting diffusion and the validity of our calculations is that the device length is much larger than the ambipolar diffusion length [1]. Although a special case where $C_{t h} \approx C_{g r}$ is feasable because of the numerical factor in equation (32), one is tempted to state that mostly $C_{t h} \ll$ $C_{g r}$. It would be interesting, however, to check experimentally whether the condition $\left(L / L_{a}\right)<5$, which would imply $C_{t h}>C_{g r}$, is still consistent with the model chosen.

Bilger et al. [2] leave no doubt that their device is operating in the insulator regime. The ratio $L / L_{a}$ is approximately 3 in their case. This implies according to equation (32) that $C_{t h} / C_{g r}=2 \cdot 5$. Unfortunately, however, no clear low frequency plateaus are found so that no unambiguous interpretation in terms of the theory presented in this paper is possible. If one follows the procedure of Bilger et al. by fitting $\left(1+\omega^{2} \tau^{2}\right)^{-1}$ curves to the experimental spectra, assuming $\tau$ independent of the injection level, low frequency plateaus are found in the insulator regime which are in fair agreement to our equation (30), i.e. these are to a large extent due to thermal noise.

Acknowledgement-The authors are greatly indebted to Prof. Alkemade for his critical reading of the manuscript.

\section{REFERENCES}

1. M. A. Lampert, Current Injection in Solids, Academic Press, New York (1970).

2. H. R. Bilger and M. Nicolet, Proc. Int. Conf. on Noise in Semiconductor Devices, Toulouse (1971).

3. D. Lee and M. Nicolet, Phys. Rev. 184, 806 (1969).

4. F. Driedonks and R. J. J. Zijlstra, Proc. Conf. on Physical Aspects of Noise in Electronic Devices, p. 95, Peregrinus, Hertfordshire, England (1968).

5. F. Driedonks, Physica 46, 291 (1970).

6. A. v.d. Ziel and K. M. van Vliet, Solid-St. Electron. 11, $508(1968)$. 